\title{
The GAPS programme with HARPS-N at TNG
}

\section{The multi-planet system KELT-6: Detection of the planet KELT-6 c and measurement of the Rossiter-McLaughlin effect for KELT- $6 b^{\star, \star \star}$}

\author{
M. Damasso ${ }^{1}$, M. Esposito ${ }^{2,3}$, V. Nascimbeni ${ }^{4,5}$, S. Desidera ${ }^{4}$, A.S. Bonomo ${ }^{1}$, A. Bieryla ${ }^{6}$, L. Malavolta ${ }^{4,5}$, K. Biazzo ${ }^{7}$, \\ A. Sozzetti ${ }^{1}$, E. Covino ${ }^{8}$, D. W. Latham ${ }^{6}$, D. Gandolfi, ${ }^{9,10}$, M. Rainer ${ }^{11}$, C. Petrovich ${ }^{12}$, K. A. Collins ${ }^{13,14}$, \\ C. Boccato ${ }^{4}$, R.U. Claudi ${ }^{4}$, R. Cosentino ${ }^{7,15}$, R. Gratton ${ }^{4}$, A.F. Lanza ${ }^{7}$, A. Maggio ${ }^{16}$, G. Micela ${ }^{16}$, E. Molinari ${ }^{15,17}$, \\ I. Pagano $^{7}$, G. Piotto ${ }^{4,5}$, E. Poretti ${ }^{11}$, R. Smareglia ${ }^{18}$, L. Di Fabrizio ${ }^{15}$, P. Giacobbe ${ }^{1}$, M. Gomez-Jimenez ${ }^{2,3}$, \\ S. Murabito ${ }^{2,3}$, M. Molinaro ${ }^{18}$, L. Affer ${ }^{16}$, M. Barbieri ${ }^{19}$, L. R. Bedin ${ }^{4}$, S. Benatti ${ }^{4}$, F. Borsa ${ }^{9}$, J. Maldonado ${ }^{16}$, \\ L. Mancini ${ }^{20,1}$, G. Scandariato ${ }^{7}$, J. Southworth ${ }^{21}$, and R. Zanmar Sanchez ${ }^{7}$
}

(Affiliations can be found after the references)

Received 19 July 2015 / Accepted 26 August 2015

\begin{abstract}
Aims. For more than 1.5 years we spectroscopically monitored the star KELT-6 (BD+31 2447), which is known to host the transiting hot-Saturn KELT- 6 b, because a previously observed long-term trend in radial velocity time series suggested that there is an outer companion.

Methods. We collected a total of 93 new spectra with the HARPS-N and TRES spectrographs. A spectroscopic transit of KELT-6 b was observed with HARPS-N, and simultaneous photometry was obtained with the IAC-80 telescope.

Results. We proved the existence of an outer planet with a mininum mass $M_{\mathrm{p}} \sin i=3.71 \pm 0.21 M_{\mathrm{Jup}}$ and a moderately eccentric orbit $(e=$ $0.21_{-0.036}^{+0.039}$ ) of period $P \sim 3.5$ years. We improved the orbital solution of KELT- $6 \mathrm{~b}$ and obtained the first measurement of the Rossiter-McLaughlin effect, showing that the planet has a likely circular, prograde, and slightly misaligned orbit with a projected spin-orbit angle of $\lambda=-36 \pm$ 11 degrees. We improved the KELT- $6 \mathrm{~b}$ transit ephemeris from photometry and provide new measurements of the stellar parameters. KELT-6 appears as an interesting case for studying the formation and evolution of multi-planet systems.
\end{abstract}

Key words. stars: individual: KELT-6 - planetary systems - techniques: radial velocities - techniques: photometric

\section{Introduction}

Analysing stellar radial velocity (RV) time series is an effective method of detecting and characterizing distant planets with orbital periods of a few years, despite the large observing time span required. To look for such companions, particularly interesting targets are stars with transiting planets, because they are an ideal laboratory for studying the architecture of multiplanet systems. In fact, the orbital geometry of the transiting planet can be described through the Rossiter-McLaughlin (RM) effect (e.g. Ohta et al. 2005) by measuring the projected spinorbit angle and spotting the direction of the motion with respect to that of the stellar rotation. Up to now, long-term trends have been observed in the RVs of a large sample of stars with and without evidence of turnover (e.g. Knutson et al. 2014). Among them is KELT-6, a late F-type, metal-poor star $(V=10.3 \mathrm{mag})$

* Based on observations made with (i) the HARPS-N spectrograph on the Italian Telescopio Nazionale Galileo (TNG), operated on the island of La Palma by the INAF - Fundacion Galileo Galilei (Spanish Observatory of Roque de los Muchachos of the IAC); (ii) the Tillinghast Reflector Echelle Spectrograph (TRES) on the 1.5-m Tillinghast telescope, located at the Smithsonian Astrophysical Observatory's Fred L. Whipple Observatory on Mt. Hopkins in Arizona; (iii) the IAC-80 telescope at the Teide Observatory (Instituto de Astrofísica de Canarias, IAC).

$\star \star$ Figure 4 and Tables 2 and 3 are available in electronic form at http://www. aanda.org hosting the transiting Saturn-mass planet KELT-6b discovered by the KELT-North survey (Collins et al. 2014, hereafter Co14). Co14 were also able to observe an unexplained residual trend in the RVs over a limited time span of 475 days. To understand the cause of this acceleration, a spectroscopic follow-up was carried out in the framework of the Global Architectures of Planetary Systems (GAPS) project ${ }^{1}$, using the HARPS-N spectrograph (resolving power $R=115000$; Cosentino et al. 2012). We also collected new RV data with the Tillinghast Reflector Echelle Spectrograph (TRES) spectrograph $(R=44$ 000; Fúresz 2008), extending the total observing time span to 1178 days.

Together with new photometric measurements, we present results that noticeably extend knowledge about the KELT-6 system.

\section{Observations and data reduction methods}

We collected 71 HARPS-N spectra (exposure $900 \mathrm{~s}$, typical signal-to-noise per pixel $S / N \sim 60$ at $5500 \AA$ ) between 2014 February 9 and 2015 July 3, 31 of which were obtained on 2015 April 11 during a transit of KELT- $6 \mathrm{~b}$ and used to study the RM effect. The Th-Ar simultaneous calibration was not used to avoid contamination by the lamp lines. The spectra and the RV measurements were reduced using the latest version (Nov. 2013) of

\footnotetext{
1 http://www . oact. inaf.it/exoit/EXO-IT/Projects/ Entries/2011/12/27\$_\$GAPS.html
} 
the HARPS-N instrument Data Reduction Software pipeline and applying a G2 mask. The measurement of the RVs is based on the weighted cross-correlation function $(\mathrm{CCF})$ method (Baranne et al. 1996; Pepe et al. 2002).

With TRES we collected 22 spectra between 2013 December 13 and 2015 May 27. They were extracted following the procedures described by Buchhave et al. (2010). The relative RVs were derived by cross-correlating the spectra against the highest S/R spectrum in the wavelength range 4050-5650 A.

Simultaneously with the RM effect measurements gathered with HARPS-N, we collected the transit light curve with the IAC-80 0.82-m telescope. Data were taken from 21:18 UT to 5:32 UT, using the CAMELOT camera $(E 2 \mathrm{~V} 2 \mathrm{k} \times 2 \mathrm{k}$ CCD; pixel scale $0.304^{\prime \prime}$; field of view $10.4^{\prime} \times 10.4^{\prime}$ ) and through a standard Bessell $R$ filter. The point spread function (PSF) was intentionally defocused to a radius of $\sim 20$ physical pixels to minimize flat-field residual errors and avoid detector saturation. The exposure time was set to $90 \mathrm{~s}$, resulting in a net cadence of $\sim 115 \mathrm{~s}$ when considering the overheads. Science frames were bias- and flat-field-corrected by standard procedures. Photometric measurements were made with the STARS $K Y$ pipeline (Nascimbeni et al. 2011, 2013). STARS $K Y$ delivered the best differential light curve of KELT-6 (i.e. the one with the least scatter) using a set of four stable comparison stars (UCAC4 604-049448, UCAC4 604-049449, UCAC4 604-049450, and UCAC4 604-049454). Unfortunately, our observations were plagued by technical problems that delayed the start time of the observations, and the telescope pointing was re-adjusted, causing an offset between the first and the second halves of the light curve, which was included as a parameter in the transit model.

\section{Stellar parameters}

The photospheric parameters were derived with different methods from the co-added HARPS-N spectrum obtained from the out-of-transit observations $(S / R \sim 380$ per pixel at $5500 \AA)$. We used the LTE code MOOG (Sneden 1973), along with atmospheric models (Kurucz 1992; Castelli \& Kurucz 2004) and iron equivalent widths (EW). Two analyses were independently performed with the main differences being the list of iron lines and the technique used to measure EWs. In one case we used ARESv2 (Sousa et al. 2015) with the automatic continuum placement set-up and the line list from Sousa et al. (2011), adapted to the 2014 version of MOOG (Dumusque et al. 2014). In the other case, the EWs of the line list from Biazzo et al. (2012) were measured by hand using the IRAF task SPLOT, and the iron abundance was determined using the 2013 version of MOOG (see Damasso et al. 2015). In both cases, the analysis was performed differentially with respect to the Sun spectrum. We also did a third independent analysis based on the method described in Gandolfi et al. (2015). We fitted the HARPS-N spectrum to a grid of theoretical models from Castelli \& Kurucz (2004), using spectral features that are sensitive to different photospheric parameters.

All the analyses gave consistent results, so we calculated their weighted averages and adopted the average of the individual uncertainties as errors. Table 1 summarizes our results. We notice that our best-fit value for $T_{\text {eff }}$ is $170 \mathrm{~K}$ higher than value adopted by Co14, which was obtained from spectral synthesis modelling with Spectroscopy Made Easy (SME; Valenti \& Piskunov 1996) using HIRES spectra. This difference could arise from the different analysis techniques rather than from the properties of the spectra, by noting that a well known bias exists
Table 1. Stellar parameters for the star KELT-6 derived from the analysis of the HARPS-N spectra and from stellar evolutionary tracks.

\begin{tabular}{cccc}
\hline \hline Parameter & This work & Co14 & Note \\
\hline$T_{\text {eff }}[\mathrm{K}]$ & $6272 \pm 61$ & $6102 \pm 43$ & \\
$\log g[\mathrm{cgs}]$ & $4.12 \pm 0.07$ & $4.074_{-0.070}^{+0.045}$ & \\
{$[\mathrm{Fe} / \mathrm{H}][\mathrm{dex}]$} & $-0.27 \pm 0.06$ & $-0.281_{-0.038}^{+0.039}$ & \\
Microturb. $\xi\left[\mathrm{km} \mathrm{s}^{-1}\right]$ & $1.49 \pm 0.1$ & $0.85(\mathrm{fixed})$ & \\
$V \sin I_{\star}\left[\mathrm{km} \mathrm{s}^{-1}\right]$ & $4.53 \pm 0.26$ & $5.0 \pm 0.5$ & $(1)$ \\
$\mu$ linear law limb-darkening coeff. & $0.48 \pm 0.14$ & & $(1)$ \\
Mass $\left[M_{\odot}\right]$ & $1.126 \pm 0.058$ & $1.085_{-0.040}^{+0.043}$ & $(2)$ \\
Radius $\left[R_{\odot}\right]$ & $1.529_{-0.137}^{+0.143}$ & $1.580_{-0.094}^{+0.160}$ & $(2)$ \\
Age $[\mathrm{Gyr}]$ & $4.90_{-0.46}^{+0.66}$ & $6.1 \pm 0.2$ & $(2)$ \\
Luminosity $\left[L_{\odot}\right]$ & $3.24 \pm 0.62$ & $3.11_{-0.39}^{+0.68}$ & $(2)$ \\
Density $\left[\mathrm{g} \mathrm{cm}^{-3}\right]$ & $0.44_{-0.10}^{+0.15}$ & $0.387_{-0.088}^{+0.068}$ & \\
\hline
\end{tabular}

Notes. Estimates from the KELT-6b discovery paper are also listed. (1) Derived from HARPS-N spectra (RM effect). (2) Matching $T_{\text {eff }}$, $[\mathrm{Fe} / \mathrm{H}]$, and $\log g$ to the Yonsei-Yale evolutionary tracks.

in some versions of SME (Torres et al. 2012), which was removed in more recent versions (Brewer et al. 2015).

The stellar mass, radius, and age were determined by comparing our measured $T_{\text {eff }}, \log g$, and $[\mathrm{Fe} / \mathrm{H}]$ with the Yonsei-Yale evolutionary tracks (Demarque et al. 2004) through the $\chi^{2}$ statistics (Santerne et al. 2011). Results are listed in Table 1. The adopted errors include an extra 5\% in mass and 3\% in radius added in quadrature to the formal errors to take systematic uncertainties into account in the stellar models (Southworth 2011). We also employed the stellar density $\rho_{\star}$ derived by Co14 as a proxy of the stellar luminosity instead of $\log g$ (e.g. Sozzetti et al. 2007). The results are fully consistent with the previous findings and not more precise. We thus adopted the parameters obtained using $\log g$, finding that KELT-6 appears to be slightly less evolved than stated by Co14.

\section{Improved transit ephemeris for KELT- 6 b}

The light curve observed with the IAC-80 telescope is shown in the upper panel of Fig. 1. It has an average photometric scatter of 0.9 mmag on a $115 \mathrm{~s}$ timescale.

Since we could not measure the out-of-transit flux of KELT-6 to properly normalize the off-transit level of our light curve, a full, detailed modelling to extract all the orbital parameters ${ }^{2}$ of KELT- $6 \mathrm{~b}$ was hampered by the unsolvable degeneracy between the normalization level and the other transit parameters. Therefore we chose to fix $R_{\star} / a, R_{\mathrm{p}} / a$, and $i$ to the best-fit values published by Co14, limiting the number of free parameters to four ${ }^{3}$. The fit was performed with the code JKTEBOP v34 (Southworth 2008), and the associated errors were derived through 10000 classical Monte Carlo iterations.

From the fit we could only determine the time of central transit $T_{\mathrm{c}}$ of KELT-6 $\mathrm{b}$. We then derived an improved ephemeris $\left(T_{\mathrm{c}}\right.$ and orbital period $P$ ) from the observed-minus-calculated

2 The fractional radii $R_{\star} / a$ and $R_{\mathrm{p}} / a$, with $R_{\star}$ being the star radius, $R_{\mathrm{p}}$ the planet radius, and $a$ the planet semi-major axis; the orbital inclination $i$; the orbital period $P$; the time of central transit $T_{0}$.

3 i.e. $T_{0}$; the linear term $u_{1}$ of a quadratic limb darkening law, with the quadratic term interpolated from the Claret \& Bloemen (2011) tables; the off-transit normalization level; an existing zero-point offset between the first and the second halves of the light curve. 

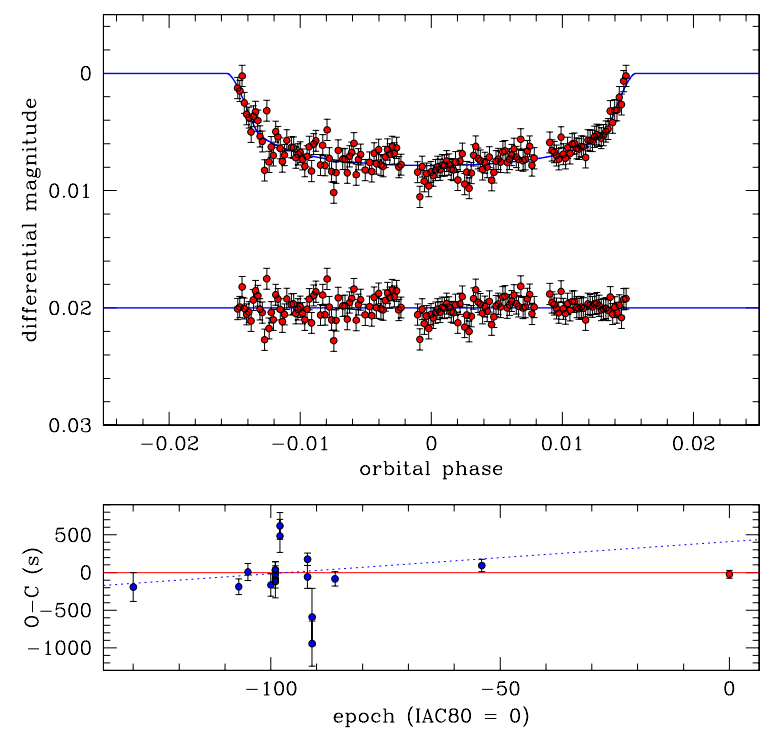

Fig. 1. Upper panel: KELT-6 b transit light curve (2014 Apr. 11, IAC-80 telescope), and residuals from the best fit model (which is plotted with a solid blue line). The root mean square of the residuals is 0.9 mmag. Lower panel: $\mathrm{O}-\mathrm{C}$ diagram including the highest-quality measurements from $\mathrm{C} 014$ plus one unpublished measurement from the KELT team (blue points on the left side) and from our IAC- 80 observation (red point). The $\mathrm{O}-\mathrm{C}$ diagram is folded on our newly determined ephemeris (solid red line), while the Co14 ephemeris is plotted with a dotted blue line.

diagram (lower panel of Fig. 1), by fitting both our measurement of $T_{\mathrm{c}}$ and the high-quality ("primary") points from $\mathrm{Co} 14$ through a weighted least-squares procedure, as well as an additional ephemeris obtained by the KELT team from an unpublished transit that occurred on 2014 February 12 . The results are shown in Table 4, with the reference epoch set on the most recent transit.

\section{Analysis of the radial velocities}

\subsection{KELT-6 c comes out}

The HARPS-N and TRES RVs collected for this work are listed in Tables 2 and 3, respectively. In our sample, we also included the RVs used in Co14 and collected by TRES and the High Resolution Echelle Spectrometer (HIRES) at the Keck telescope, while we excluded the first 26 HARPS-N measurements taken during the night of the KELT- $6 \mathrm{~b}$ transit. The RV time series shows a long-term modulation with a clear turnover and a semi-amplitude higher than that of the short-period signal due to KELT-6 b (Fig. 2). KELT-6 appears to be a quiet star $\left(\left\langle\log \left(R_{\mathrm{HK}}^{\prime}\right)\right\rangle=-4.992, \sigma_{\log \left(R_{\mathrm{HK}}^{\prime}\right)}=0.021\right)$, without evidence in the $\log \left(R_{\mathrm{HK}}^{\prime}\right)$ data for a modulation ascribable to an activity cycle (Fig. 4, upper plot). Besides the $\log \left(R_{\mathrm{HK}}^{\prime}\right)$ index, we calculated the CCF bisector span (BIS) from the HARPS-N spectra, and we found that no significant correlations exist over the timespan of the HARPS-N measurements between these datasets and the RVs with the orbital solution of KELT-6 b removed (Fig. 4, middle and lower plots). This evidence supports the hypothesis that the observed RV long-term variations are not due to the stellar activity but to an outer companion, which we name KELT-6 c and for which the data cover almost one complete orbit. This scenario is further strengthened by looking, for example, at the empirical calibrations of Santos et al. (2000) (see Eq. (2) and Fig. 6 therein) that, for a star like KELT-6, predict a
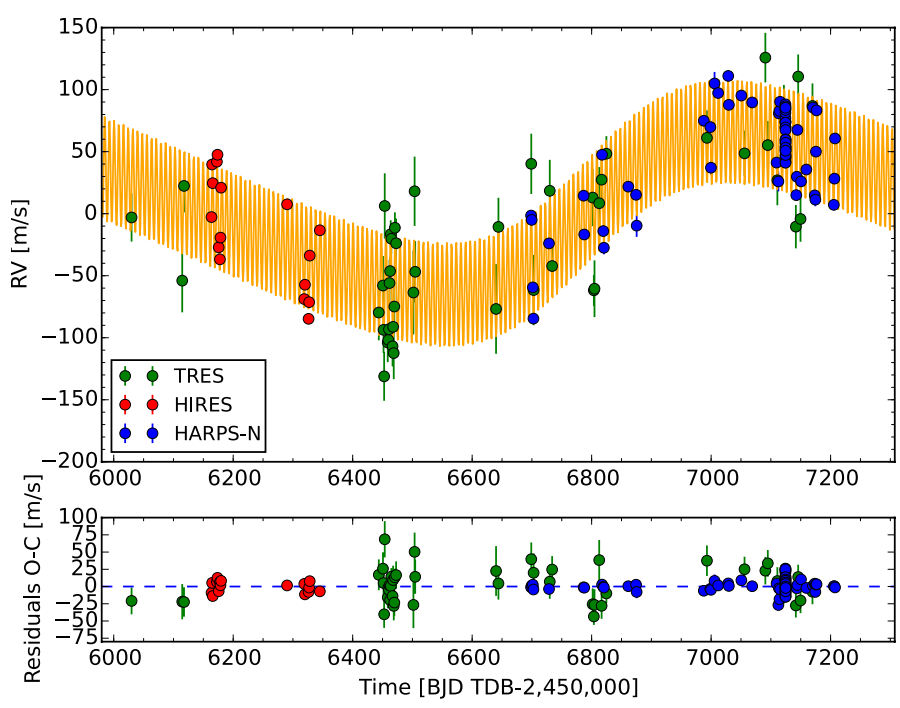

Fig. 2. Upper panel: radial velocity time series of KELT-6. The $1 \sigma$ errorbars are over-plotted to each data point. When they are not visible, this means they are smaller than the symbol size. Over-plotted is our best two-planet Keplerian model (orange line), calculated from the bestfit orbital parameters of Table 4. Lower panel: residuals of the best-fit two-planet model.

RV dispersion due to activity-related phenomena at the level of $\sim 10 \mathrm{~m} \mathrm{~s}^{-1}$, while our measured semi-amplitude is $\sim 3-5$ times higher $\left(K \sim 66 \mathrm{~m} \mathrm{~s}^{-1}\right)$ once the orbital solution of KELT-6b is removed. Orbital parameters and uncertainties for the KELT planets were determined with a Bayesian differential evolution Markov chain Monte Carlo analysis (e.g. Desidera et al. 2014). We adopted a two-planet Keplerian model with sixteen free parameters $^{4}$, assuming for KELT-6 $\mathrm{b}$ Gaussian priors on $T_{0}$ and $P$ based on the new ephemeris. Fitted and derived parameters are listed in Table 4. The best-fit model is shown in Fig. 2, and the residuals show a dispersion of $\sim 17 \mathrm{~m} \mathrm{~s}^{-1}$, which reduces to $9.5 \mathrm{~m} \mathrm{~s}^{-1}$ when considering only the more precise HIRES and HARPS-N measurements. This fact agrees nicely with the level of the activity-related jitter predicted by Santos et al. (2000).

KELT-6 c appears to be massive $\left(M_{\mathrm{p}} \sin i=3.71 \pm 0.21 M_{\text {Jup }}\right)$ and moves on a moderately eccentric orbit (significant at the $\sim 5 \sigma$ level) with period $P \sim 3.5$ years. Residuals of the twoplanet model do not reveal evidence of any trend of having a third companion (Fig. 2). Our results suggest that KELT-6 b has a likely circular orbit, with the eccentricity $e=0.029_{-0.013}^{+0.016} \mathrm{com}$ patible with zero within $\sim 2.45 \sigma$ (Lucy $\&$ Sweeney 1971 ). A reanalysis of the data done by the KELT team corrected the eccentricity to the value $e=0.058 \pm 0.034$, which is in accordance with our measurement.

\subsection{The Rossiter-McLaughlin effect for KELT-6 b}

The RV time series covering the transit of KELT- $6 \mathrm{~b}$ was analysed using the numerical model and the least-squares fitting algorithm described in Covino et al. (2013) and Esposito et al. (2014). Four parameters were set free ${ }^{5}$, while all other relevant

4 The central transit epoch $T_{0}$, the orbital period $P$, the RV semiamplitude $K, \sqrt{e} \cos \omega$, and $\sqrt{e} \sin \omega$ of both planets KELT-6b and KELT-6c ( $e$ and $\omega$ being the eccentricity and the argument of periastron); a jitter term and a RV offset for each of the different datasets.

$5 \gamma$ : barycentric RV at mid-transit epoch; $\lambda$ : projected angle between the planetary orbital axis and the stellar spin axis; $V \sin I_{*}$ : projected stellar rotational velocity; $\mu$ : limb-darkening coefficient of a linear law. 
Table 4. Orbital and physical parameters for KELT-6 b and KELT-6 c.

\begin{tabular}{|c|c|c|}
\hline Parameter & KELT-6b & KELT-6 c \\
\hline \multicolumn{3}{|l|}{ Primary transit analysis } \\
\hline$T_{\mathrm{c}}\left[\mathrm{BJD}_{\mathrm{TDB}}-2450000\right]$ & $7124.50954 \pm 5.7 \times 10^{-4}$ & \\
\hline$P$ [days] & $7.845582 \pm 7 \times 10^{-6}$ & \\
\hline$\left(R_{\mathrm{p}} / R_{\mathrm{S}}\right)^{a}$ & $0.077613_{-0.0009}^{+0.0010}$ & \\
\hline$b^{a, b}$ & $0.20_{-0.13}^{+0.0 .14}$ & \\
\hline$i^{a, c}[\mathrm{deg}]$ & $88.81_{-0.91}^{-0.13}$ & \\
\hline \multicolumn{3}{|l|}{$R V$ analysis } \\
\hline$P^{d}$ [days] & $7.8455821 \pm 7 \times 10^{-6}$ & $1276_{-67}^{+81}$ \\
\hline$K^{d}\left[\mathrm{~m} \mathrm{~s}^{-1}\right]$ & $41.8 \pm 1.1$ & $65.7_{-2.4}^{+2.6}$ \\
\hline$e^{e}$ & $0.029_{-0.013}^{+0.016}$ & $0.21_{-0.036}^{+0.44}$ \\
\hline$(\sqrt{e} \sin \omega)^{d}$ & $-0.083_{-0.086}^{+0.120}$ & $-0.454 \pm 0.042$ \\
\hline$(\sqrt{e} \cos \omega)^{d}$ & $0.126_{-0.046}^{+0.035}$ & $-0.011 \pm 0.071$ \\
\hline$\omega^{e}[\mathrm{deg}]$ & $308_{-272}^{-3.040}$ & $268.7 \pm 8.8$ \\
\hline$T_{\mathrm{c}}{ }^{d}\left[\mathrm{BJD}_{\mathrm{TDB}}-2450000\right]$ & $7124.50954 \pm 5.8 \times 10^{-4}$ & $7432_{-32}^{+39}$ \\
\hline$\gamma_{\mathrm{TRES}}{ }^{d}\left[\mathrm{~m} \mathrm{~s}^{-1}\right]$ & \multicolumn{2}{|l|}{$46.3 \pm 3.8$} \\
\hline$\gamma_{\mathrm{HIRES}}{ }^{d}\left[\mathrm{~m} \mathrm{~s}^{-1}\right]$ & \multicolumn{2}{|l|}{$22.1 \pm 8.5$} \\
\hline$\gamma_{\text {HARPS-N }}^{d}\left[\mathrm{~m} \mathrm{~s}^{-1}\right]$ & \multicolumn{2}{|l|}{$1128.9 \pm 3.0$} \\
\hline$\left(\text { jitter }_{\text {TRES }}\right)^{d}\left[\mathrm{~m} \mathrm{~s}^{-1}\right]$ & \multicolumn{2}{|l|}{$14.2 \pm 4.5$} \\
\hline$\left(\text { jitter }_{\mathrm{HIRES}}\right)^{d}\left[\mathrm{~m} \mathrm{~s}^{-1}\right]$ & \multicolumn{2}{|l|}{$8.1_{-1.8}^{+2.3}$} \\
\hline$\left(\text { jitter }_{\text {HARPS-N }}\right)^{d}\left[\mathrm{~m} \mathrm{~s}^{-1}\right]$ & \multicolumn{2}{|l|}{$2.2 \pm 1.2$} \\
\hline$\left(M_{\mathrm{p}} \sin i\right)^{e, f}\left[M_{\mathrm{Jup}}\right]$ & $0.442 \pm 0.019$ & $3.71 \pm 0.21$ \\
\hline$R_{\mathrm{p}}^{g}\left[R_{\mathrm{Jup}}\right]$ & $1.18 \pm 0.11$ & \\
\hline$a_{\mathrm{p}}^{h}[\mathrm{AU}]$ & $0.080 \pm 0.001$ & $2.39 \pm 0.11$ \\
\hline$\gamma_{\mathrm{RM}}^{i}\left[\mathrm{~m} \mathrm{~s}^{-1}\right]$ & $1190.1 \pm 1.4$ & \\
\hline$\lambda^{i}[\mathrm{deg}]$ & $-36 \pm 11$ & \\
\hline
\end{tabular}

Notes. ${ }^{(a)}$ Co14. ${ }^{(b)}$ Impact parameter. ${ }^{(c)}$ Inclination of the orbital plane. ${ }^{(d)}$ Fitted. ${ }^{(e)}$ Derived. ${ }^{(f)}$ For KELT-6 b this corresponds to the real mass of the planet, derived by assuming the best estimate of the inclination angle of the orbital plane provided by Co14. ${ }^{(g)}$ Derived from the transit parameter $R_{\mathrm{p}} / R_{\mathrm{s}}$ in Co14 and our value of $R_{\mathrm{s}}$ through a Monte-Carlo analysis. ${ }^{(h)}$ Derived from the third Kepler's law through a Monte-Carlo analysis, using our updated values for the stellar mass and planetary orbital periods. ${ }^{(i)}$ Fitted (Rossiter-McLaughlin effect).

parameters were kept fixed to the values derived from the spectroscopic and photometric analyses. The best-fit values are reported in Tables 1 and 4, together with their uncertainties derived by means of a bootstrapping method. The best-fit RM model is shown in Fig. 3. Results show that KELT-6 b moves in a prograde orbit and is slightly misaligned with respect to the stellar spin axis with a projected spin-orbit angle $\lambda=-36^{\circ} \pm 11^{\circ}$. The lack of enough RV data in the pre-transit phase means that a value of $\lambda$ closer to zero cannot be completely ruled out, but our solution comes with a lower $\chi^{2}$ than for the case of $\lambda$ fixed to 0 (1.39 vs. 1.89$)$.

\section{Discussion}

KELT-6 joins a small number of host stars with a transiting planet and a measured RM effect for which there is evidence of outer companions. To our knowledge they are HAT-P-13, HAT-P-17, and WASP-8 (Knutson et al. 2014), the last two hosting a transiting planet with the orbital period close to that of KELT-6b (respectively, $P=10.3$ and 8.2 days) and with WASP- 8 being misaligned $\left(\lambda=-123.3_{-4.4}^{+3.4}\right)^{6}$. Our results allow

\footnotetext{
6 See http://www.astro.keele.ac.uk/jkt/tepcat/ rossiter.html
}

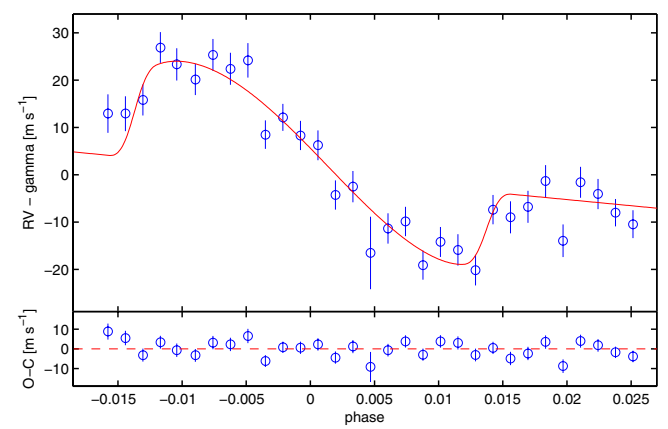

Fig. 3. HARPS-N RV time series spanning the transit of KELT-6 b occurred on 2015 April 11 (open blue circles) and our best-fit model of the Rossiter-McLaughlin effect (red solid line).

the description of the KELT-6 system architecture in some detail, and considerations can be made about its dynamical evolution and stability.

We tested the coplanar high-eccentricity migration hypothesis (CHEM, Petrovich 2015a) as a possible mechanism to explain the current orbital elements of KELT-6. CHEM explores the possibility that hot Jupiters are formed through secular gravitational interactions with an outer planet on an initial eccentric orbit, and then circularized by tides with the host star at periastron. The mechanism describes the planetary migration as occurring on nearly the same plane where the planets formed, assuming a low mutual inclination of the orbital planes $\left(\lesssim 20^{\circ}\right)$, and it predicts that hot Jupiters should have distant and more massive companions with moderately high eccentricities $(e \sim 0.2-0.5)$. CHEM reproduces the observed architecture of KELT-6, provided that the inner planet, initially with $e \gtrsim 0.5$, started migration inside $\sim 1 \mathrm{au}$, and there was an initial moderate mutual inclination of $\sim 10^{\circ}-20^{\circ}$ to account for the measured angle $\lambda$. This suggests that the current mutual inclination of the orbital planes could be lying in this range. We note that the system should be old enough for the orbit of KELT- $6 \mathrm{~b}$ to have been circularized. In fact, the circularization time with the current system parameters is about $0.45 \mathrm{Gyr}$, assuming a modified tidal quality factor $Q_{\mathrm{p}}^{\prime}=10^{5}$ for the planet (Lainey et al. 2009), where most of the tidal dissipation is expected to have occurred in the case of an initially eccentric orbit (see Jackson et al. 2008). We speculate that the initial conditions required by CHEM to explain this system could be the result of a preceding phase of planet-planet scattering. A system with more than two planets in nearly circular and coplanar orbits at $\gtrsim 1$ au might have become unstable, losing planets by ejections and leading to eccentricity excitation and moderate change (by a factor up to $\sim 2$ ) in the semi-major axis of the inner planet (Jurić \& Tremaine 2008; Chatterjee et al. 2008).

We investigated the dynamical stability of the KELT-6 system by using the empirical relation (17) in Petrovich (2015b), who studied the final results of the evolution on long timescales of two-planet systems with arbitrary eccentricities and mutual inclinations against either ejections or collisions with the host star. We found that the relation is satisfied well, implying a stability preserved on a timescale of a few Myr (i.e. the range of validity of Petrovich's formula), as expected for a system that is already $\sim 5$ Gyr old.

Acknowledgements. GAPS acknowledges support from INAF through the "Progetti Premiali" funding scheme of the Italian Ministry of Education, University, and Research. M.D. acknowledges support from INAF-OATo through grant \#35/2014, and thanks the Astronomical Observatory of the Autonomous Region of the Aosta Valley for its support with computing 
infrastructure. IRAF is distributed by the National Optical Astronomy Observatories, operated by the Association of Universities for Research in Astronomy, Inc., under cooperative agreement with the National Science Foundation. We made use of the SIMBAD database and VizieR catalogue access tool, operated at the CDS, Strasbourg, France.

\section{References}

Baranne, A., Queloz, D., Mayor, M., et al. 1996, A\&AS, 119, 373 Biazzo, K., D’Orazi, V., Desidera, S., et al. 2012, MNRAS, 427, 2905

Brewer, J. M., Fischer, D. A., Basu, S., Valenti, J. A., \& Piskunov, N. 2015, ApJ, 805,126

Buchhave, L. A., Bakos, G. Á., Hartman, J. D., et al. 2010, ApJ, 720, 1118

Castelli, F., \& Kurucz, R. L. 2004, ArXiv e-prints [arXiv:astro-ph/0405087]

Chatterjee, S., Ford, E. B., Matsumura, S., \& Rasio, F. A. 2008, ApJ, 686, 580

Claret, A., \& Bloemen, S. 2011, A\&A, 529, A75

Collins, K. A., Eastman, J. D., Beatty, T. G., et al. 2014, AJ, 147, 39

Cosentino, R., Lovis, C., Pepe, F., et al. 2012, in SPIE Conf. Ser., 8446

Covino, E., Esposito, M., Barbieri, M., et al. 2013, A\&A, 554, A28

Damasso, M., Biazzo, K., Bonomo, A. S., et al. 2015, A\&A, 575, A111

Demarque, P., Woo, J.-H., Kim, Y.-C., \& Yi, S. K. 2004, ApJS, 155, 667

Desidera, S., Bonomo, A. S., Claudi, R. U., et al. 2014, A\&A, 567, L6

Dumusque, X., Bonomo, A. S., Haywood, R. D., et al. 2014, ApJ, 789, 154

Esposito, M., Covino, E., Mancini, L., et al. 2014, A\&A, 564, L13

Fúresz, G. 2008, Ph.D. Thesis, Univ. of Szeged, Hungary

Gandolfi, D., Parviainen, H., Deeg, H. J., et al. 2015, A\&A, 576, A11

Jackson, B., Greenberg, R., \& Barnes, R. 2008, ApJ, 678, 1396

Jurić, M., \& Tremaine, S. 2008, ApJ, 686, 603

Knutson, H. A., Fulton, B. J., Montet, B. T., et al. 2014, ApJ, 785, 126

Kurucz, R. L. 1992, in The Stellar Populations of Galaxies: Proc. IAU Symp., 149,225

Lainey, V., Arlot, J.-E., Karatekin, Ö., \& van Hoolst, T. 2009, Nature, 459, 957

Lucy, L. B., \& Sweeney, M. A. 1971, AJ, 76, 544

Nascimbeni, V., Piotto, G., Bedin, L. R., \& Damasso, M. 2011, A\&A, 527, A85

Nascimbeni, V., Cunial, A., Murabito, S., et al. 2013, A\&A, 549, A30

Ohta, Y., Taruya, A., \& Suto, Y. 2005, ApJ, 622, 1118

Pepe, F., Mayor, M., Galland, F., et al. 2002, A\&A, 388, 632

Petrovich, C. 2015a, ApJ, 805, 75

Petrovich, C. 2015b, ApJ, 808, 120

Santerne, A., Díaz, R. F., Bouchy, F., et al. 2011, A\&A, 528, A63

Santos, N. C., Mayor, M., Naef, D., et al. 2000, A\&A, 361, 265

Sneden, C. 1973, ApJ, 184, 839

Sousa, S. G., Santos, N. C., Adibekyan, V. Z., Delgado Mena, E., \& Israelian, G. 2015, A\&A, 577, A67

Sousa, S. G., Santos, N. C., Israelian, G., Mayor, M., \& Udry, S. 2011, 533, A141

Southworth, J. 2008, MNRAS, 386, 1644

Southworth, J. 2011, MNRAS, 417, 2166

Sozzetti, A., Torres, G., Charbonneau, D., et al. 2007, ApJ, 664, 1190
Torres, G., Fischer, D. A., Sozzetti, A., et al. 2012, ApJ, 757, 161

Valenti, J. A., \& Piskunov, N. 1996, A\&AS, 118, 595

1 INAF-Osservatorio Astrofisico di Torino, via Osservatorio 20, 10025, Pino Torinese, Italy

e-mail: damasso@oato.inaf.it

2 Instituto de Astrofísica de Canarias, C/Via Láctea S/N, 38200 La Laguna, Tenerife, Spain

3 Departamento de Astrofísica, Universidad de La Laguna, 38205 La Laguna, Tenerife, Spain

4 INAF-Osservatorio Astronomico di Padova, Vicolo dell'Osservatorio 5, 35122 Padova, Italy

5 Dip. di Fisica e Astronomia Galileo Galilei - Università di Padova, Vicolo dell'Osservatorio 2, 35122 Padova, Italy

6 Harvard-Smithsonian Center for Astrophysics, Cambridge, MA 02138, USA

7 INAF-Osservatorio Astrofisico di Catania, via S.Sofia 78, 95123, Catania, Italy

8 INAF-Osservatorio Astronomico di Capodimonte, Salita Moiariello 16, 80131 Napoli, Italy

9 Dipartimento di Fisica, Universitá di Torino, via P. Giuria 1, 10125 Torino, Italy

10 Landessternwarte Königstuhl, Zentrum für Astronomie der Universität Heidelberg, Königstuhl 12, 69117 Heidelberg, Germany

11 INAF-Osservatorio Astronomico di Brera, via E. Bianchi 46, 23807 Merate (LC), Italy

12 Department of Astrophysical Sciences, Princeton University, Ivy Lane, Princeton, NJ 08544, USA

13 Department of Physics and Astronomy, Vanderbilt University, Nashville, TN 37235, USA

14 Department of Physics and Astronomy, University of Louisville, Louisville, KY 40292, USA

15 Fundación Galileo Galilei - INAF, Rambla José Ana Fernandez Pérez 7, 38712 Breña Baja, TF, Spain

16 INAF-Osservatorio Astronomico di Palermo, Piazza del Parlamento, 1, 90134 Palermo, Italy

17 INAF-IASF Milano, via Bassini 15, 20133 Milano, Italy

18 INAF-Osservatorio Astronomico di Trieste, via Tiepolo 11, 34143 Trieste, Italy

19 Universidad de Atacama, Departamento de Fisica, Copayapu 485, Copiapo, Chile

20 Max Planck Institute for Astronomy, Königstuhl 17, 69117 Heidelberg, Germany

21 Astrophysics Group, Keele University, Staffordshire, ST5 5BG, UK

Page 6 is available in the electronic edition of the journal at http://wwW . aanda.org 
Table 2. Sample list of all the radial velocities (RV) measured for KELT-6 with the HARPS-N spectrograph in the framework of the GAPS programme.

\begin{tabular}{|c|c|c|c|c|c|}
\hline $\begin{array}{c}\text { Time } \\
\left(\mathrm{BJD}_{\mathrm{UTC}}-2450000\right)\end{array}$ & $\begin{array}{c}\mathrm{RV} \\
\left(\mathrm{m} \mathrm{s}^{-1}\right)\end{array}$ & $\begin{array}{l}\text { RV err. } \\
\left(\mathrm{m} \mathrm{s}^{-1}\right)\end{array}$ & $\begin{array}{c}\text { BIS } \\
\left(\mathrm{m} \mathrm{s}^{-1}\right)\end{array}$ & $\log \left(R_{\mathrm{HK}}^{\prime}\right)$ & $\log \left(R_{\mathrm{HK}}^{\prime}\right)$ err. \\
\hline 6698.624617 & 1127.6 & 3.7 & 85.6 & -4.953 & 0.017 \\
\hline 6699.598118 & 1124.1 & 2.7 & 92.6 & -4.995 & 0.011 \\
\hline 6701.572178 & 1069.7 & 3.2 & 76.1 & -4.993 & 0.016 \\
\hline 6702.578027 & 1044.5 & 3.6 & 83.2 & -4.967 & 0.015 \\
\hline 6728.651408 & 1105.1 & 3.8 & 91.5 & -5.020 & 0.021 \\
\hline 6786.609623 & 1143.6 & 4.6 & 103.4 & -4.952 & 0.027 \\
\hline 6787.608024 & 1112.3 & 3.9 & 101.6 & -4.983 & 0.023 \\
\hline 6817.482897 & 1176.6 & 2.7 & 83.9 & -5.022 & 0.013 \\
\hline 6819.529437 & 1115.1 & 3.6 & 78.6 & -4.987 & 0.019 \\
\hline 6820.503665 & 1101.6 & 5.3 & 78.4 & -5.035 & 0.040 \\
\hline 6861.396206 & 1150.9 & 2.5 & 87.9 & -5.001 & 0.011 \\
\hline 6874.367191 & 1144.3 & 3.7 & 94.1 & -4.996 & 0.020 \\
\hline 6875.369909 & 1119.5 & 9.1 & 67.0 & -4.975 & 0.076 \\
\hline 6987.775868 & 1204.0 & 3.6 & 86.8 & -4.968 & 0.019 \\
\hline 6998.737459 & 1198.9 & 5.5 & 81.9 & -4.950 & 0.032 \\
\hline 6999.772079 & 1166.2 & 5.7 & 107.6 & -5.021 & 0.044 \\
\hline 7005.756862 & 1234.1 & 9.3 & 71.0 & -5.034 & 0.086 \\
\hline 7011.773901 & 1226.4 & 2.7 & 90.9 & -4.997 & 0.012 \\
\hline 7028.700777 & 1240.1 & 3.5 & 101.0 & -5.015 & 0.019 \\
\hline 7029.691968 & 1216.8 & 3.5 & 87.4 & -5.001 & 0.018 \\
\hline 7050.675470 & 1224.3 & 4.8 & 84.4 & -4.985 & 0.030 \\
\hline 7068.780530 & 1218.8 & 4.7 & 109.7 & -4.971 & 0.028 \\
\hline 7109.622389 & 1170.3 & 5.0 & 87.8 & -5.000 & 0.031 \\
\hline 7112.604654 & 1155.1 & 8.0 & 78.7 & -4.996 & 0.069 \\
\hline 7113.520997 & 1209.9 & 3.7 & 86.7 & -4.977 & 0.021 \\
\hline 7114.443438 & 1212.0 & 5.0 & 74.6 & -4.960 & 0.032 \\
\hline 7115.451479 & 1219.3 & 2.6 & 90.4 & -5.003 & 0.012 \\
\hline 7124.384992 & 1203.2 & 4.1 & 89.8 & -4.958 & 0.024 \\
\hline 7124.395594 & 1203.2 & 3.7 & 95.3 & -4.983 & 0.022 \\
\hline 7124.406149 & 1206.1 & 3.3 & 101.8 & -4.989 & 0.018 \\
\hline 7124.416785 & 1217.2 & 3.2 & 109.7 & -5.013 & 0.019 \\
\hline 7124.427595 & 1213.6 & 3.4 & 97.6 & -4.994 & 0.020 \\
\hline 7124.438266 & 1210.4 & 3.3 & 85.0 & -4.992 & 0.018 \\
\hline 7124.448937 & 1215.6 & 3.4 & 78.9 & -4.968 & 0.018 \\
\hline 7124.459481 & 1212.7 & 3.4 & 99.1 & -5.013 & 0.020 \\
\hline 7124.470175 & 1214.5 & 3.6 & 86.5 & -5.006 & 0.022 \\
\hline 7124.481309 & 1198.8 & 3.0 & 82.7 & -5.013 & 0.016 \\
\hline 7124.491679 & 1202.4 & 2.9 & 94.5 & -4.994 & 0.014 \\
\hline 7124.502581 & 1198.6 & 3.1 & 80.4 & -5.001 & 0.016 \\
\hline 7124.513125 & 1196.5 & 3.2 & 85.3 & -4.987 & 0.016 \\
\hline 7124.524155 & 1186.0 & 3.1 & 83.6 & -5.006 & 0.016 \\
\hline 7124.534618 & 1187.8 & 3.3 & 84.4 & -4.988 & 0.018 \\
\hline 7124.543668 & 1173.8 & 7.7 & 97.4 & -4.956 & 0.056 \\
\hline 7124.556156 & 1179.0 & 3.2 & 91.7 & -5.013 & 0.018 \\
\hline 7124.566665 & 1180.4 & 3.1 & 98.1 & -5.005 & 0.017 \\
\hline 7124.577441 & 1171.2 & 3.1 & 92.9 & -5.015 & 0.017 \\
\hline 7124.587949 & 1176.1 & 3.2 & 89.6 & -4.997 & 0.017 \\
\hline 7124.599130 & 1174.4 & 3.3 & 72.1 & -5.001 & 0.019 \\
\hline 7124.609685 & 1170.2 & 3.2 & 91.4 & -5.011 & 0.018 \\
\hline 7124.620229 & 1182.9 & 3.1 & 103.7 & -4.998 & 0.017 \\
\hline 7124.631085 & 1181.3 & 3.4 & 83.3 & -4.996 & 0.018 \\
\hline 7124.641640 & 1183.5 & 3.4 & 69.9 & -5.002 & 0.018 \\
\hline 7124.652496 & 1189.0 & 3.4 & 91.8 & -4.976 & 0.017 \\
\hline 7124.663202 & 1176.4 & 3.5 & 81.3 & -4.982 & 0.018 \\
\hline 7124.673908 & 1188.7 & 3.3 & 83.9 & -4.985 & 0.016 \\
\hline 7124.684347 & 1186.2 & 3.2 & 89.2 & -4.987 & 0.017 \\
\hline 7124.695354 & 1182.3 & 2.9 & 101.9 & -4.995 & 0.015 \\
\hline 7124.705932 & 1179.9 & 2.9 & 101.8 & -4.980 & 0.015 \\
\hline 7142.537170 & 1144.0 & 3.6 & 89.5 & -4.990 & 0.021 \\
\hline 7143.470202 & 1158.9 & 3.4 & 86.3 & -5.018 & 0.020 \\
\hline 7144.467594 & 1196.6 & 3.8 & 84.4 & -5.003 & 0.023 \\
\hline 7150.499394 & 1155.1 & 4.6 & 90.2 & -5.004 & 0.026 \\
\hline 7159.414630 & 1164.7 & 3.2 & 99.1 & -5.024 & 0.019 \\
\hline 7170.469914 & 1214.6 & 2.7 & 83.9 & -5.010 & 0.012 \\
\hline 7173.479806 & 1143.8 & 5.2 & 112.2 & -4.961 & 0.033 \\
\hline 7174.499116 & 1140.4 & 5.3 & 91.6 & -4.937 & 0.032 \\
\hline 7175.462458 & 1179.1 & 2.7 & 99.2 & -4.996 & 0.013 \\
\hline 7176.476235 & 1212.3 & 3.8 & 82.5 & -4.994 & 0.021 \\
\hline 7205.414495 & 1136.2 & 2.5 & 90.2 & -5.000 & 0.011 \\
\hline 7206.421521 & 1157.3 & 3.1 & 80.1 & -4.968 & 0.014 \\
\hline 7207.432852 & 1189.7 & 3.8 & 87.3 & -4.952 & 0.021 \\
\hline
\end{tabular}

Notes. Also indicated are the bisector velocity span (BIS) and the activity index $\log \left(R_{\mathrm{HK}}^{\prime}\right)$. The BIS are those derived by the data reduction pipeline of HARPS-N with uncertainties assumed to be twice those on the radial velocities.
Table 3. New relative radial velocities of KELT-6 measured with the TRES spectrograph and used for the first time in this work.

\begin{tabular}{ccc}
\hline \hline $\begin{array}{c}\text { Time } \\
\left(\mathrm{BJD}_{\mathrm{UTC}}-2450000\right)\end{array}$ & $\begin{array}{c}\text { RV } \\
\left(\mathrm{m} \mathrm{s}^{-1}\right)\end{array}$ & $\begin{array}{c}\text { RV error } \\
\left(\mathrm{m} \mathrm{s}^{-1}\right)\end{array}$ \\
\hline 6640.021901 & -30.50 & 36.12 \\
6643.998845 & 35.66 & 23.47 \\
6698.953413 & 86.48 & 24.29 \\
6702.883586 & -15.28 & 28.33 \\
6729.914621 & 64.77 & 24.93 \\
6733.902128 & 4.10 & 19.52 \\
6801.709729 & 59.28 & 22.98 \\
6803.711379 & -15.48 & 12.28 \\
6804.800386 & -14.19 & 22.87 \\
6812.690753 & 54.72 & 29.16 \\
6816.685639 & 73.70 & 18.88 \\
6824.685293 & 94.65 & 14.15 \\
6993.029954 & 107.42 & 22.14 \\
7055.946535 & 94.92 & 18.22 \\
7090.798944 & 172.10 & 19.99 \\
7094.908058 & 101.59 & 19.32 \\
7110.916771 & 73.25 & 20.13 \\
7121.849369 & 133.02 & 17.07 \\
7141.862950 & 35.84 & 17.49 \\
7145.767451 & 156.82 & 17.82 \\
7149.696112 & 42.03 & 18.32 \\
7169.684005 & 133.14 & 18.14 \\
\hline & &
\end{tabular}
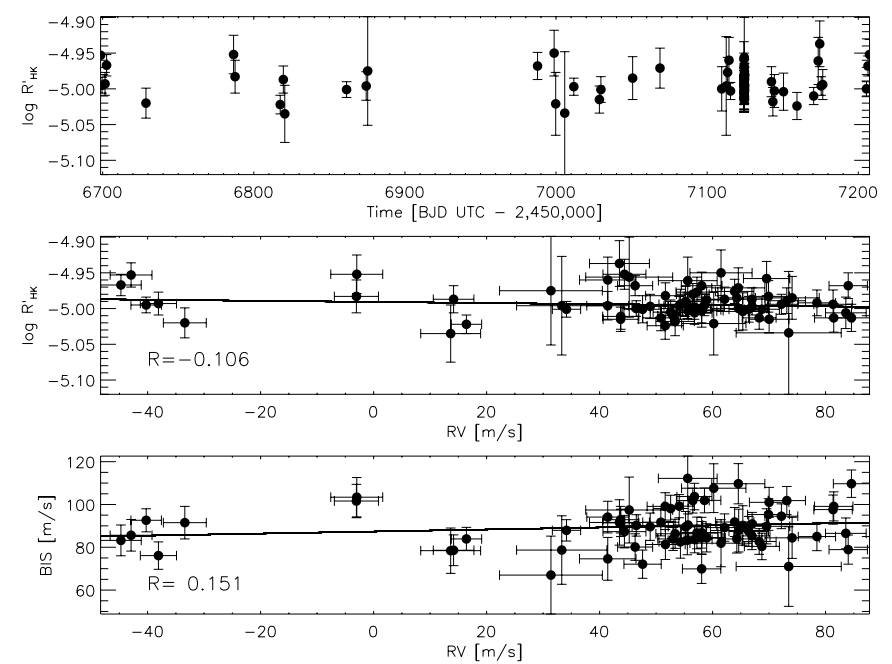

Fig. 4. Upper panel: time series of the $\log \left(R_{\mathrm{HK}}^{\prime}\right)$ chromospheric index as measured from HARPS-N spectra. Middle and lower panels: correlation analysis between the RV residuals, obtained by removing the Keplerian signal due to KELT-6 b from the original HARPS-N dataset, and two indicators of stellar activity derived from the HARPS-N spectra: the $\log \left(R_{\mathrm{HK}}^{\prime}\right)$ chromospheric index and the CCF bisector span. Overplotted are the values of Spearman's rank correlation coefficients, which show the absence of significant correlations and support the hypothesis that the origin of the observed RV long-term variations is Keplerian. 